\title{
ANALYSIS OF SIGNAL FORMATION AND CONVERSION IN HYBRID RADIO OVER FIBRE NETWORK
}

\author{
Oleksandr Iashchuk, Iuliia Iashchuk, Konstantin Sunduchkov \\ National Technical University of Ukraine "Kiev Polytechnic Institute" \\ Institute of Telecommunication Systems \\ Kiev, Ukraine, Industrial lane, 2 \\ Ph.: +380 (95) 421-43-70.E-mail: yaas@bigmir.net
}

\begin{abstract}
Calculation methods of the signal formation parameters at the central station and of selective conversion in mobile terminal receiver of the vehicle moving at speed of 200-300 km per h are described. Architecture features of radio over fibre networks to operate without handover between base stations lead to additional requirements for transmitting and receiving signal methods in the network. The solution of this problem will provide high-rate services (up to 12 Mbit per second) for subscribers moving on the highway or high-speed railway using hybrid radio over fibre network. It allows providing services of any content at any time. Objective: calculation methods development of the signal formation and conversion parameters. Signal is a set of services in the form of OFDM-symbols, the total bandwidth of which is much higher than $1 \mathrm{GHz}$.
\end{abstract}

Keywords: radio over fibre network, signal formation of services set, selective conversion in mobile terminal receiver

\section{Introduction}

Modern development of telecommunications caused to the need to ensure services availability to users anywhere and anytime. Acceptable speed of mobile users has increased to 200-300 km per h. This scenario is relevant for high-speed roads and railways. However, mobile terminal (MT) problems associated with the MT transition from one base station (BS) to another (handover) limit the speed values to $100-150 \mathrm{~km}$ per $\mathrm{h}$ [1]. In this case, the data transfer rate is not exceeding 100 Mbit per second.

Hybrid radio over fibre (RoF) technology allows achieving the required speed of mobile subscribers' access to multimedia resources. RoF technology combines high capacity of wired networks and portability of wireless networks [2]. Hybrid RoF architecture includes service providers, central station (CS), optical links, BS and MT [3]. Topology options and signal transmission methods define the architecture of the whole network. In [4] BS is connected to CSusing star topology. It is proposed to unite several cells in virtual zones to artificially increase cell. Handover management is carried out in CA. Subscriber movement is limited at $100 \mathrm{~km}$ per h. In [5] research the star topology is also used and moving cells method is proposed. The necessary services are moving with MT. The need of signal and MT moving synchronization complicates CS functions and limits the speed to $130 \mathrm{~km}$ per $\mathrm{h}$. To achieve high network capability of network it is mandatory to use millimetre waves that can accommodate a wide band signal.

RoF network architecture with high capacity and ability to serve customers moving at speed of 300 $\mathrm{km}$ per $\mathrm{h}$ is shown in [3].

This architecture has the following features, which reduce the deploying cost in comparison with similar products:

- Tree topology of connection between CSand base stations. Tree topology reduces the required total length of the fibre optic cable in the network compared to star topology;

- Simplified function of BS. It significantly reduces the whole network cost. It is required a lot of BS because of signal attenuation in the millimetre wave range. It leads to cell size decreasing.

This functional architecture allows providing services to customers at high bit rate. The principal feature is that all BS work in the same band and all BS receive all network services and transmit it in their cell.

Features of this network architecture perform demands to signal transmission in CSand receiving of signal in user's MT.

New ways of signal formation in CS and its selective conversion in user's MT receiver methods as well as multi-criteria mathematical model for calculating the parameters of these methods are proposed in $[6,7]$. Solution is shown using the integral preference criterion. However, this solution is partial and allows finding the best option among all randomly generated, but not all valid options. 
The purpose of the article is to propose calculation methods for signal formation and selective conversion parameters in hybrid RoF network. Solution differs from other known existing by using of absolute Pareto criterion and the possibility of best option finding from all valid.

\section{Network Description}

Traditionally telecommunication network has two directions of communication: downlink and uplink channels. Downlink channel is responsible for information transmission from network to user, and the uplink channel is responsible for from user to network information transmission. An example of using such hybrid RoF network architecture in the downlink channel is shown as a challenge to provide mobile communications along railway line from Moscow to St. Petersburg. The real task raw data are shown on Figure 1.

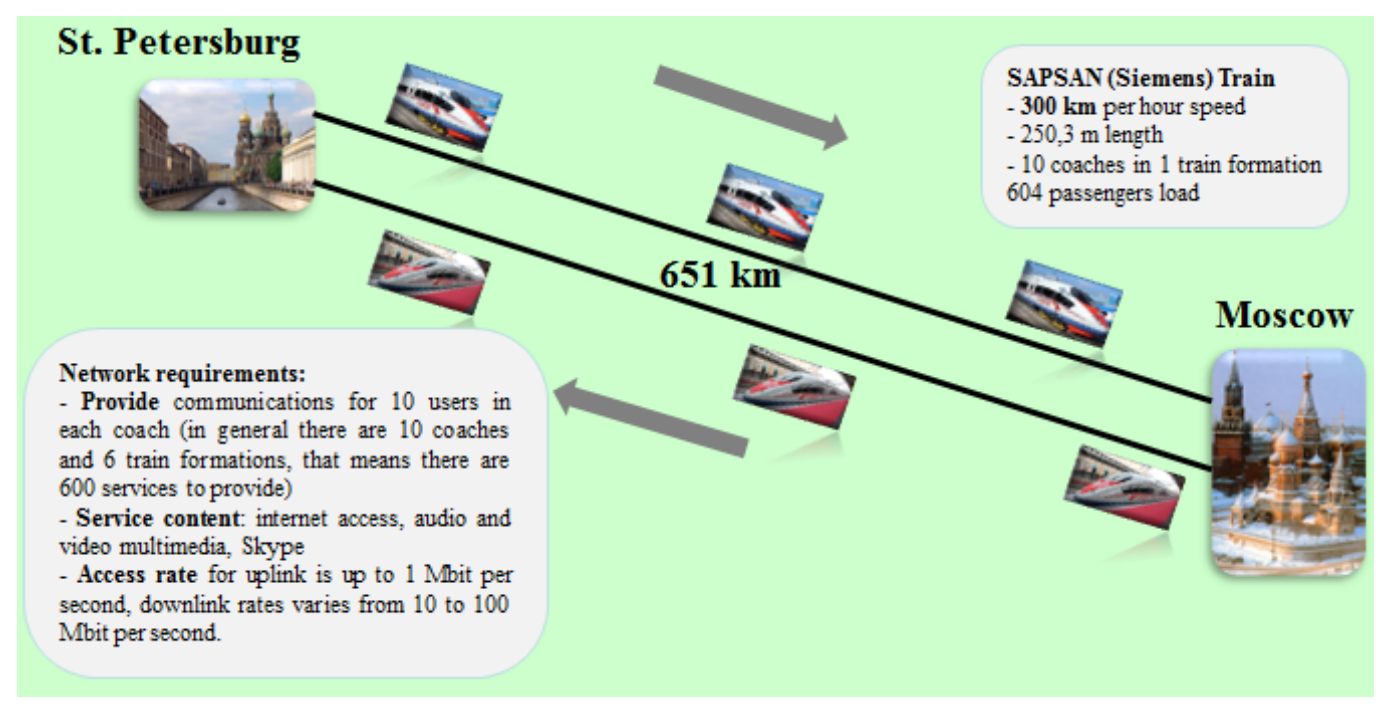

Figure 1. Raw data for task to ensure mobile communications along railway route from Moscow to St. Petersburg

Internet access for customers is organized via two Wi-Fi access points, installed in each coach. Downlink channel scheme using hybrid RoF is shown on Figure 2.

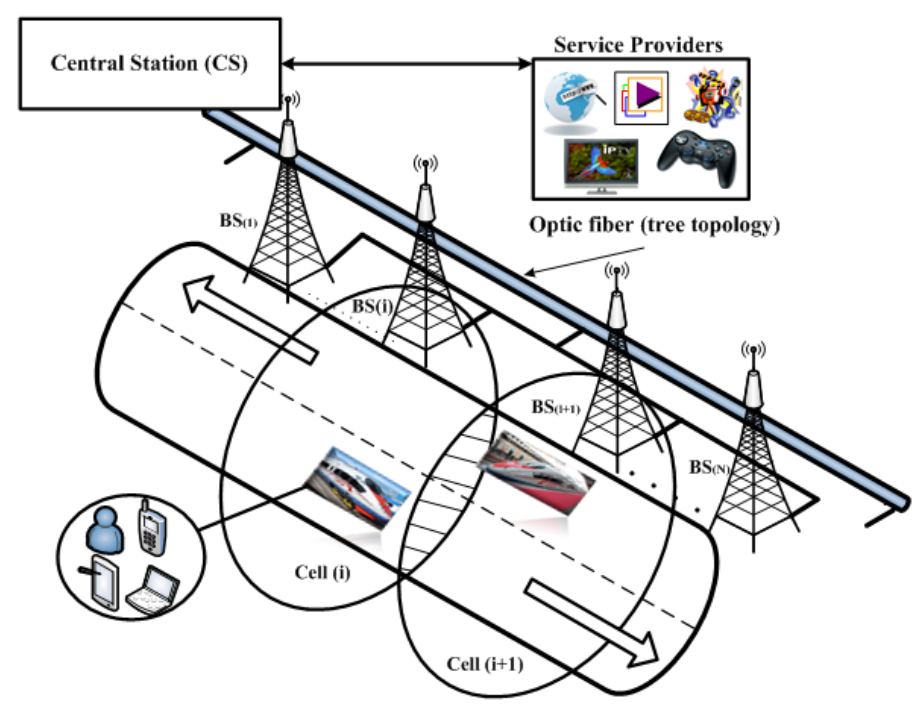

Figure 2. Hybrid mobile network architecture that serves users on high-speed

CS receives required services from service providers. It multiplexes all subscribers' services (at current time) to one stream. Aggregate signal after optical modulator is sent to all BS in tree topology. 
Two optical fibres fit to BS, the first with information signal and the second with a reference beam of light. The wavelength difference determines the carrier frequency in the millimetre range. The BS photodiode converts the difference between two lights into electromagnetic emission. BS receives all network services and transmits them in cell.

Network capacity for downlink equals to 10-100 (Mbit per second) • 600 (subscribers) = 6-60 (Gb per second) that will take 1-10 GHz band using 64-QAM signal.

Uplink channel works on mobile WiMax technology:

- WiMax transmitter with capacity of 100 Mbit per second (100 subscribers multiply on 1Mbit per second) is set in the middle of train;

- Receivers (the BS) with capacity of 200 Mbit per second are set along railway on $25 \mathrm{~km}$ distance (for the simultaneous two trains moving in one cell).

To ensure the handover it is necessary to implement diameter of cells overlapping so that train transit time in the overlap is greater (with margin) then time required for WiMax system handover performing.

Spread spectrum multi-carrier technology based on OFDM technology is used for signal formation scheme in CS to provide high system capacity and frequency spectrum effective usage.

\section{Mathematical Models of Service Set Signal Formation and Conversion}

\subsection{Mathematical model of signal formation in the central station}

It is assumed that the multi service RoF network provides two types of services: low-rate services with rate $V_{1}$ [bit per second], and high-rate services with rate $V_{2}$ [bit per second]. Total amount of services provided is $v_{1}$ first type number of services and $v_{1}$ second type number of services, bringing the total number of services in the $K=v_{1}+v_{2}$. $K$ equals the maximum possible number of subscribers in the system.

All $K$ services should be divided into groups of 64, 128, 256 or 512 services, depending on the modulators set used at the transmitting end. Parameters $a_{1}, a_{2}, a_{3}, a_{4}$ corresponds to the group number of $64,128,254,512$ services with $V_{1}$ rate; $b_{1}, b_{2}, b_{3}, b_{4}$ corresponds to the group number of 64, 128, 254,512 services respectively with $V_{2}$ rate. It is considered for simplicity that QAM-64 is used in OFDM-symbol with 64 subcarriers, QAM-128 in OFDM-symbol with 128 subcarriers, etc. Each OFDM-symbol is a group containing up to 64, 128, 256 or 512 services using QAM-64, QAM-128, QAM-256, QAM-512 modulation, respectively. OFDM-symbol amount determines the number of groups in the system $\left(N_{g r}\right)$. Monthly fee for the $V_{1}$ service equals to $A$ [units], and for the $V_{2}$ service it equals to $B$ [units].

Parameter $K_{d}$ is used as the demand for high-rate service ratio. It is defined as

$$
K_{d}=\frac{v_{2}^{d}}{v_{2}} \text {, }
$$

where $v_{2}^{d}$ is the second type services amount, which is used by subscribers, $v_{2}$ is the total amount of second type services in the network.

If we denote the parameter $k$ as ratio between the second type services amount to the total number of services in the system $k=\frac{v_{2}}{v_{1}+v_{2}}$, then the dependence of $K_{d}$ from $k$ (or it percentage, $\left.k[\%]=\frac{v_{2}}{v_{1}+v_{2}} \cdot 100 \%\right)$ is determined statistically and refers to the input data.

Each type of modulator has its nominal cost characterizing the technological development level, and $c_{1}, c_{2}, c_{3}, c_{4}$ [units] corresponds to QAM-64, QAM-128, QAM-256, QAM-512 modulators cost for the service at $V_{1}$ rate [bit per second]; $d_{1}, d_{2}, d_{3}, d_{4}$ [units] corresponds to QAM-64, QAM-128, QAM-256, QAM-512 modulators cost for the service at $V_{2}$ rate [bit per second]. Obviously, more complex is modulator, more expensive it is. 
Input data for task solution are the following:

$V_{1}, V_{2}$ - bit rate of low-speed and high-speed services, respectively, [bit per second];

$A, B$ - monthly services fee of the first and second type, respectively, [units];

$K$ - total number of services in the system;

$c_{1}, c_{2}, c_{3}, c_{4}$ - modulators cost for services at $V_{1}$ rate, [units];

$d_{1}, d_{2}, d_{3}, d_{4}-$ modulators cost for services at $V_{2}$ rate, [units];

$m$ - proportionality coefficient;

$K_{d}(k)$ - dependence of demand parameter from ratio between the second type services amount to the total number of services in the system.

Parameters to be found:

$a_{1}, a_{2}, a_{3}, a_{4}$ - number of groups of $64,128,254,512$ services with $V_{1}$ rate [bit per second];

$b_{1}, b_{2}, b_{3}, b_{4}$ - number of groups of $64,128,254,512$ services with $V_{2}$ rate [bit per second].

Following quality indicators have to be used for unknown parameters:

$D=A \cdot v_{1}+K_{d} \cdot B \cdot v_{2}$ [units per month] $\rightarrow$ max - the monthly income from services provision to subscribers;

$K_{d} \rightarrow$ max- demand parameter;

$N_{g r}=\sum_{i}\left(a_{i}+b_{i}\right) \rightarrow$ min- number of service groups (OFDM-symbols);

$C=\sum_{i} m \cdot\left(a_{i} \cdot c_{i}+b_{i} \cdot d_{i}\right)$ [units] $\rightarrow$ min -total cost of the system that is proportional to the modulators cost in the system;

$E=\frac{\Delta f}{R}$ [Hz/bits per second $] \rightarrow$ min-spectral usage efficiency, where $\Delta f[\mathrm{~Hz}]-$ bandwidth required to transmit all services, $R$ [bit per second] - the total bit rate of all services.

\subsection{Mathematical model of selective signal conversion for services set in mobile terminal}

The solution of the signal formation task is the input data for finding the parameters of the selective signal conversion of service set in the MT receiver.

The state-of-the-art level that expresses the possibility of band-pass filters to allocate bandwidth on particular carrier also is included to input data. The ratio of bands width allocation to the carrier frequency is supposed to be no less that $t_{1}$, but no more that $t_{2}$. In this case, we assume that the ideal value $t_{i d}$ stays between $t_{1}$ and $t_{2}\left(t_{1} \leq t_{i d} \leq t_{2}\right)$.

Input data for task solution are the following:

$a_{1}, a_{2}, a_{3}, a_{4}$ - number of groups of $64,128,254,512$ services with $V_{1}$ rate [bit per second];

$b_{1}, b_{2}, b_{3}, b_{4}$ - number of groups of $64,128,254,512$ services with $V_{2}$ rate [bit per second];

$t_{1}, t_{i d}, t_{2}$ - parameters showing state-of-the-art level that expresses the possibility of band-pass filters to allocate bandwidth on particular carrier.

Definition: frequency area is the frequency domain with $\Delta f_{a}$ band on intermediate frequency, which is transmitted to digital signal processing area, and which includes one or more service groups.

Figure 3 shows the diagram of signal bandwidth conversion when signal is received.

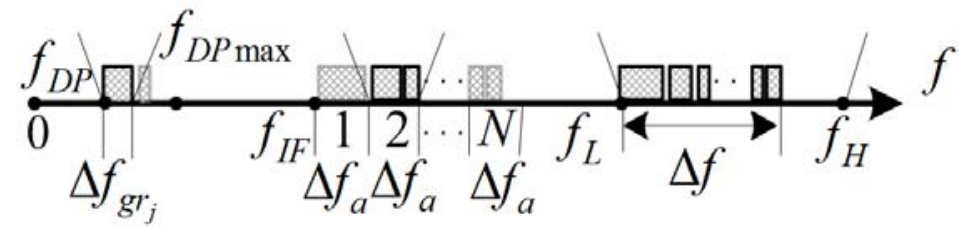

Figure 3. The settings of signal receiver 
Parameters to be found:

1) $f_{L}, f_{H}$ - the lowest and highest operating frequency of $\Delta f$ band which is transmitted to intermediate frequency not depending on input signal structure. This allows using same type equipment in the conversion first stage for all options. It is equal to the maximum possible bandwidth of signal frequency $\max \{\Delta f\}$.

2) $\Delta f_{a}, N, f_{I F}$ - frequency area band, the number of areas required for overlapping all service groups, and the lowest intermediate frequency.

3) $f_{D P}$ is the lowest spectrum frequency in area of digital processing (DP). It is determined on the basis of following inequality $f_{D P}+\Delta f_{a}<f_{D P \max }$.

The optimal values searching of these parameters include the objective function that consists of following quality indicators:

$N \rightarrow$ min- area number minimizing, and therefore the filter number to separate all groups;

$E_{f}=\left(\Delta f_{a} \cdot N-\Delta f\right)[\mathrm{Hz}] \rightarrow$ min - inefficient spectrum usage minimizing. Inefficient spectrum usage arises by partitioning groups into areas because of the OFDM-symbol feature that if it is broken it before digital processing, then original service signals restoration fails. Consequently, one group cannot be divided and placed in two areas at the same time;

$\Psi=\frac{\sum_{i} \Psi_{i}}{N} \rightarrow$ min, where $\Psi_{i}=\left|\frac{\Delta f_{a}}{f_{I F}+(i-1) \cdot \Delta f_{a}}-t_{i d}\right|, i=1 . . N-$ shows how the ratio of the band area to the intermediate frequency rate at which it is allocated differs from $t_{i d}$. As different versions will contain a variable number of parameters $\Psi_{i}$, then to estimate them together it is necessary to minimize their average value. With the known value of the frequency band $\Delta f_{a}$ occupied by area, this option allows to select the intermediate frequency $f_{I F}$ on which the band allocation $\Delta f_{a}$ will be the most technically simplified;

$$
d[\mathrm{~m}] \rightarrow \text { max - to maximize the BS cell size ( } d \text { is inversely to } \Delta f_{a}: d \sim \frac{1}{\Delta f_{a}} \text { ). }
$$

\section{Mathematical Models Solution Methods}

\subsection{The solution method of signal formation mathematical model}

For the signal formation problem it is necessary to solve two subtasks sequentially:

1. To find the ratio between number of two types of services to maximize profits and at the same time with the maximum use of all services by subscribers (maximization of demand parameter for highrate services).

2. To find division of services into groups (what modulators have to be used) for which the total number of groups is minimized, the price of the system is minimized, spectral efficiency is maximized.

To solve the first subtask it is necessary to find quality index $D$ and demand parameter $K_{d}$. To solve the second subtask it is necessary to find number of groups $N_{g r}$, total cost of the system $C$, spectral usage efficiency $E$. Each task is solved separately below.

\subsubsection{Determining ratio between numbers of two types of services}

Let us suppose that the price of the first type service $A=1$, price of second type service $B=4$, and there are $K=2000$ services in the system. For clarification and simplicity of presentation we will use the following dependence of the demand parameter: $K_{d}(k)=1-k$. In this manner the demand parameter decreases in direct proportion to the increase of second type services. If the second type services part is $30 \%(k=0,3)$, only $70 \%\left(K_{b}(k=0,3)=0,7\right)$ of the total amount of services will be in demand.

Dependence of the normalized quality parameters $D^{n}$ and $K_{d}$ for given input data on the number of the second type services $v_{2}$ is shown on Figure 4. 


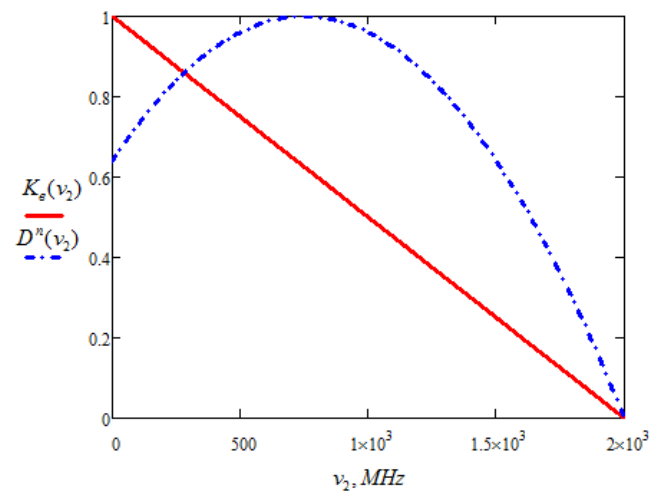

Figure 4. Dependence of normalized functions $K_{6}\left(v_{2}\right)$ and $D^{n}\left(v_{2}\right)$

It is impossible to reach maximum of these both functions at the same time. It is assumed that the solution is value of $v_{2}$, where $D^{n}(k)$ reaches its maximum. This value is $v_{2}=750$.

\subsubsection{Determining the types of modulators used to split signal into groups}

Division of the first type service does not affect the division of the second type services and vice versa. These are two separate tasks, each of which needs to be solved.

Values [units] for various types of modulators equal to $c_{1}=1, c_{2}=2, c_{3}=5, c_{4}=15$, $d_{1}=4, d_{2}=8, d_{3}=20, d_{4}=60$. The proportionality coefficient $m=3$.

Focus on quality indicators $E$ and $N_{g r}$ does not require compromise between them, as they are achieved by the use of modulation with the highest positioning. Therefore, it can be argued that it is sufficient to consider only one of these parameters, for example $N_{g r}$. It requires finding a compromise with the total cost quality parameter $C=\sum_{i} m \cdot\left(a_{i} \cdot c_{i}+b_{i} \cdot d_{i}\right)$ [units].

Let us find the structure of the modulators for the second type services (high-rate services). The task is discrete-there is a certain(finite) number of used modulators sets, each of which corresponds to a certain parameter values of $N_{g r}$ and $C$ [units].The number of groups cannot be set less than $N_{g r \min }=2$. One group (modulator) is not enough to place 750 services, because the maximum value of services in one group is 512 (usingQAM-512). The maximum number of groups using modulators with the lowest point of positioning (QAM-64) equals to $N_{g r \max }=\frac{750}{64} \approx 12$.

For each valid value of $N_{g r}$ it is necessary to find such modulators structure for which $C$ is minimum. Figure 5 shows the calculated values.

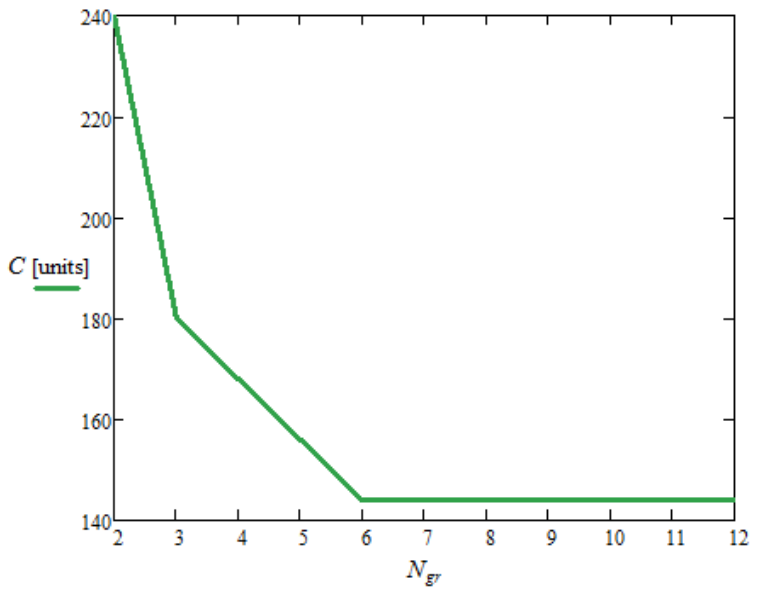

Figure 5. System price depending on the number of groups 
For options $N_{g r}>6$ the number of groups increases meanwhile system price remains fixed. These options are worse than $N_{g r}=6$. Points $\left(N_{g r}, C\right)$ for which $N_{g r}<7$ form a set of Pareto-optimal solutions. This is the set of optimal points obtained by unconditional optimisation. Further decision has to be made based on the standard criteria introduction, for example the minimax criterion of preference. Let us normalize the values of $C$ and $N_{g r}$ (relative to their maximum values $C_{\max }=240, N_{g r} \max =6$ from

Figure 5). For each option we find the maximum value $\max \left\{N_{g r}{ }^{n}, C^{n}\right\}$ of normalized quality parameters.

The minimum value among the values of $\max \left\{N_{g r}{ }^{n}, C^{n}\right\}$ will match to the option that is optimal by minimax criterion. For this case it equals to $N_{g r}=4: 2$ modulators QAM-256, 2 modulators QAM-128; $C=3 \cdot(20 \cdot 2+8 \cdot 2)=168[$ units $]$. The result is shown in Table 1 .

Table 1. Decision based on the minimax criterion

\begin{tabular}{|c|c|c|c|c|}
\hline$N_{g r}$ & $C,[$ units $]$ & $N_{g r}{ }^{n}$ & $C^{n}$ & $\max \left\{N_{g r}{ }^{n}, C^{n}\right\}$ \\
\hline 2 & 240 & 0,33 & 1 & 1 \\
\hline 3 & 180 & 0,5 & 0,75 & 0,75 \\
\hline 4 & 168 & 0,67 & 0,7 & 0,7 \\
\hline 5 & 156 & 0,83 & 0,65 & 0,83 \\
\hline 6 & 144 & 1 & 0,6 & 1 \\
\hline
\end{tabular}

Likewise, the composition of modulators for the first type services can be found.

\subsection{The solution method of selective signal conversion mathematical model}

For the problem of selective signal conversion it is necessary to solve two sub-tasks sequentially:

1. To find frequency band of areas and the number of areas so that the number of frequency bands is minimized, the frequency efficiency and the coverage area of one BSis maximized.

2. To find the intermediate frequency used in the selective conversion, so that the technical implementation coefficient of band pass filters is minimal.

\subsubsection{Determining number and frequency band of areas}

For this subtask the following input data is taken:

$a_{1}=2 ; a_{2}=1 ; a_{3}=3 ; a_{4}=1 ; b_{1}=0 ; b_{2}=0 ; b_{3}=2 ; b_{4}=0 ; t_{1}=0,05 ; t_{i d}=0,1 ; t_{2}=0,15$.

Quality parameters $N, 1 / d$ and following limitations are used to find the Pareto-optimal set of parameter options:

$\left(\Delta f_{a} \cdot N-\Delta f\right) \geq 0$ - the total bandwidth of the signal after split into zones is not less than the band of all services groups;

$\Delta f_{a} \leq 1 \mathrm{GHz}$ - the frequency band of area is less than $1 \mathrm{GHz}$ - limit of normal digital signal processing on the second intermediate frequency;

$\Delta f_{a} \geq \max \left\{\Delta f_{g r}\right\}$ - the frequency band of area is bigger than the maximum bandwidth of services group for chosen option of services distribution - any of the existing services groups should be fully located in one area, so then not to violate the integrity of one OFDM-symbol (service group);

$N$ - positive integer;

$\Delta f_{a}$ - frequency band multiple to32 $\mathrm{MHz}$ (discrete values of group area with increment equal to the lowest allowed band of services group are used, namely the group with64servicesat 3Mbitper s and QAM-64 modulation).

Dependence of $\Delta f_{a}(N) \quad$ according $\quad$ to restrictions $\quad$ on $\Delta f_{a} \quad\left(\Delta f_{a} \leq 1 \mathrm{GHz} \quad\right.$ and $\left.\Delta f_{a} \geq \max \left\{\Delta f_{g r}\right\}=384 \mathrm{MHz}\right)$ is shown on Figure 6 . 


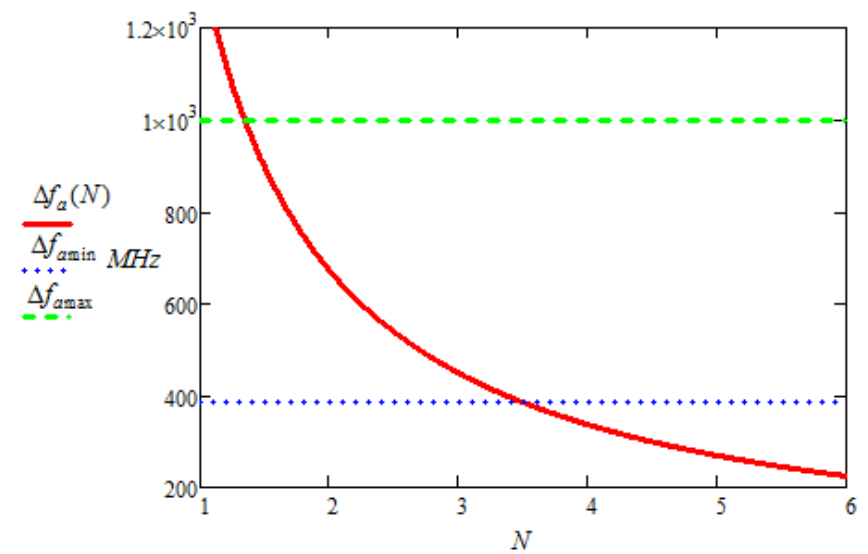

Figure 6. Dependence of the area frequency bands $\Delta f_{a}(N)$ on their number within the minimum and maximum $\Delta f_{a}$ value

According to Figure 6, to achieve the simultaneous minimization of both $N$ and $\Delta f_{a}$ values is not possible. Reducing of one parameter results in increasing of another.

Additionally it is necessary to take into account the limitation on the positive integrity of quality parameter $N$. Let us define its permissible value. $N=1$ is invalid value since then $\Delta f_{a}>1 \mathrm{GHz}$. All other values are valid (but it does not mean that they are included to Pareto-optimal set - it is shown below).

The minimum values $\Delta f_{a}$ for each of the possible $N$ values with the positive integrity limitation were found ( $\Delta f_{a}$ must be multiple to $32 \mathrm{MHz}$ ). The obtained values of $N, \Delta f_{a}$ taking into account all the limitations are shown in Figure 7.

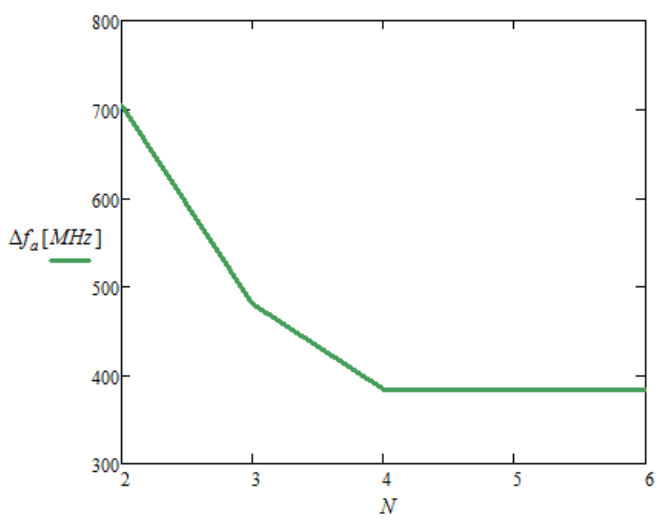

Figure 7. The obtained values of $N, \Delta f_{a}$ taking into account all the limitations

For $N>4$ options the number of frequency bands increases meanwhile band is fixed. These options are far worse than the option with $N=4$. Points $\left(N, \Delta f_{a}\right)$ where $N<5$ form a set of Paretooptimal solutions. This is the set of optimal points obtained by unconditional optimisation.

Let us find solution among the Pareto-optimal set by the minimax criterion. The values of quality parameters are normalized by the maximum values and for each of the solutions the maximum normalized value of the parameter is found. Solution by the minimax criterion is the option where the selected value is minimal (Table 2).

Table 2. Finding the optimal option from Pareto-optimal set by the minimax criterion

\begin{tabular}{|c|c|c|c|c|}
\hline$N$ & $\Delta f_{a},[M H z]$ & $N^{n}$ & $\Delta f_{a}^{n}$ & $\max \left\{N^{n}, \Delta f_{a}^{n}\right\}$ \\
\hline 2 & 704 & 0,5 & 1 & 1 \\
\hline 3 & 480 & 0,75 & 0,68 & 0,94 \\
\hline 4 & 384 & 1 & 0,55 & 1 \\
\hline
\end{tabular}


The optimum point is ( $\left.N=3, \Delta f_{a}=480 \mathrm{MHz}\right)$ according to the minimax criterion. This solution is an optimal solution of this subtask.

\subsubsection{Determining the intermediate frequency}

The quality parameter $\Psi$ for the previously found values equals to:

$\Psi=\frac{\sum_{i} \Psi_{i}}{N}=\frac{\Psi_{1}+\Psi_{2}+\Psi_{3}}{3}=\frac{1}{3}\left(\left|\frac{480}{f_{I F}}-0,01\right|+\left|\frac{480}{f_{I F}+480}-0,01\right|+\left|\frac{480}{f_{I F}+2 \cdot 480}-0,01\right|\right) \rightarrow$ min.

A graph of the function $\Psi\left(f_{I F}\right)$ is shown on Figure 8 .

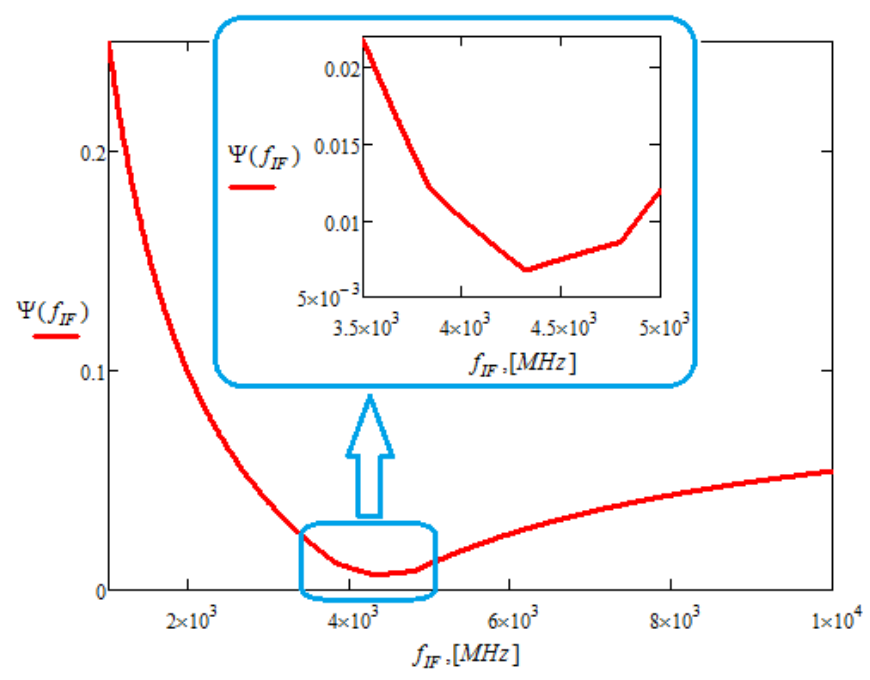

Figure 8. Dependence $\Psi\left(f_{I F}\right)$

Minimum value of $\Psi\left(f_{I F}\right)$ function is achieved at $f_{I F}=4320 \mathrm{MHz}$ point. Thus, all parameters of selective conversion were found:

$N=3, \Delta f_{a}=480 \mathrm{MHz}, f_{I F}=4320 \mathrm{MHz}$.

\section{Conclusions}

1. Method for finding the parameters of services signal formation at the central station of radio over fibre hybrid network is proposed. Rules how to determine the optimal ratio between the two types of services and optimal set of modulators for usage in the system are described.

2. Method for finding the parameters of selective conversion of all services signal in the receiver of mobile terminal is proposed. Procedure how to determine the number of frequency areas, their bandwidth and inter mediate frequency is described.

Methods differ from the existing by ability to find the solution from all valid options. It is achieved by using both unconditional Pareto optimality criterion and conditional minimax criterion.

\section{References}

1. Pleros, N., Vyrsokinos, K., Tsagkaris, K., Tselikas, N. (2009). A 60 GHz Radio-Over-Fiber Network Architecture for Seamless Communication with High Mobility. Journal of Light Wave Technology, 27(12), 1957-1967.

2. Ng’oma, A. (2009). Radio-over-Fiber Systems for Multi-Gbps Wireless Communication. In A. Ng'oma, M. Sauer (Eds.), Communications and Photonics Conference and Exhibition (ACP), vol. 7632 (pp. 1-10). 
3. Il'chenko, M.E., Sunduchkov, K.S., Volkov, S.E. et al. (2008). Interactive heterogeneous telecommunication system $4 \mathrm{G}$ with wireless access in the millimeter range for the multi media services provisioning to mobile subscribers. Communication, 7-8, 28-32. (In Russian)

4. Kim, H. B., Emmelmann, M., Rathke, B., Wolisz, A. (2005). Radio over Fiber Network Architecture for Road Vehicle Communication Systems. In Proceedings of IEEE Vehicular Technology Conference VTC 2005-Spring, 2005 IEEE 61st, Vol. 5 (pp. 2920-2924).

5. Lannoo, B., Colle, D., Pickavet, M., Demeester, P. (2003). Radio over Fiber Technique for Multimedia Train Environment. NOC, pp. 99-106.

6. Sunduchkov, K. S., Tykhonenko, I. I., Iashchuk, O. S. (2012). Formation of services set and parameters of OFDM-symbols in the distributed mobile communication network. Communication, 2 (98), 22-26. (In Russian)

7. Iashchuk, O. S., Sunduchkov, K. S. (2012). Mathematical model of selective conversion in the vehicle mobile terminal receiver. Radio Electronic and Computer Systems, 1(53), 125-131. (In Russian) 